\title{
Optimal resource allocation of the servicing companies in the field of housing and public utilities on the basis of the theory of multiphase queueing systems
}

\author{
Rustam Khayrullin ${ }^{1, *}$ \\ ${ }^{1}$ Moscow State University of Civil Engineering, Yaroslavskoe shosse, 26, Moscow, 129337, Russia
}

\begin{abstract}
The mathematical model describing technological process of functioning of the servicing companies in the sphere of housing sector and communal services is offered. The model allows you to manage the resources of serving companies in the housing sector in order to improve the quality of service. The problem statement and the solution of a problem of rational distribution of resources of the service company on the example of functioning of the center of maintenance of the measuring equipment used in the sphere of housing and communal services is given. The service center is modeled as a multiphase queueing system, which includes single-phase Queuing systems with limited and unlimited queue length. Used in the paper a method of sequential linearization allows to solve tasks of control production capacity of multiphase queueing systems for the simplest incoming flows with exponential service of requirements and free streams of requirements that are describing by means of simulation modeling methods. The software was developed. The results of applying the method of consistent linearization and corresponding software to the problem of optimal redistribution of production capacity of the center for maintenance of measuring equipment used in the sphere of housing and communal services are presented.
\end{abstract}

\section{Introduction}

The problem of improving the quality of services is relevant in the field of housing and communal services (housing) and construction [1-3] is important actual problem. Methods of mathematical modeling of servicing systems based on the Theory of Queuing Systems (QS), including Theory of Multidimensional Queuing Systems (MQS) are widely used for its investigation [4-6]. In the present work the decision of a problem of rational distribution of resources of the servicing company on an example of service of the control and Measuring Equipment (ME) applied in the sphere of housing sector and communal services is given.

\footnotetext{
Corresponding author: zrk@nm.ru
} 


\section{The mathematical model of the center of technical maintenance of measuring equipment}

The maintenance center of ME consists of Dispatcher Point (DP) and complex, including a Diagnostic Center (DC), Repair Shop (RS) and Fine Tuning Workshop (FTW) (Figure1). DP - distributes the incoming flow of requests for servicing of ME intensity in DC, RS and FTW. The values of probabilities $p_{i j}$ calculated by means of statistical methods and the intensity value $\lambda$ of incoming stream are shown next to the arrows in the Figure 1.

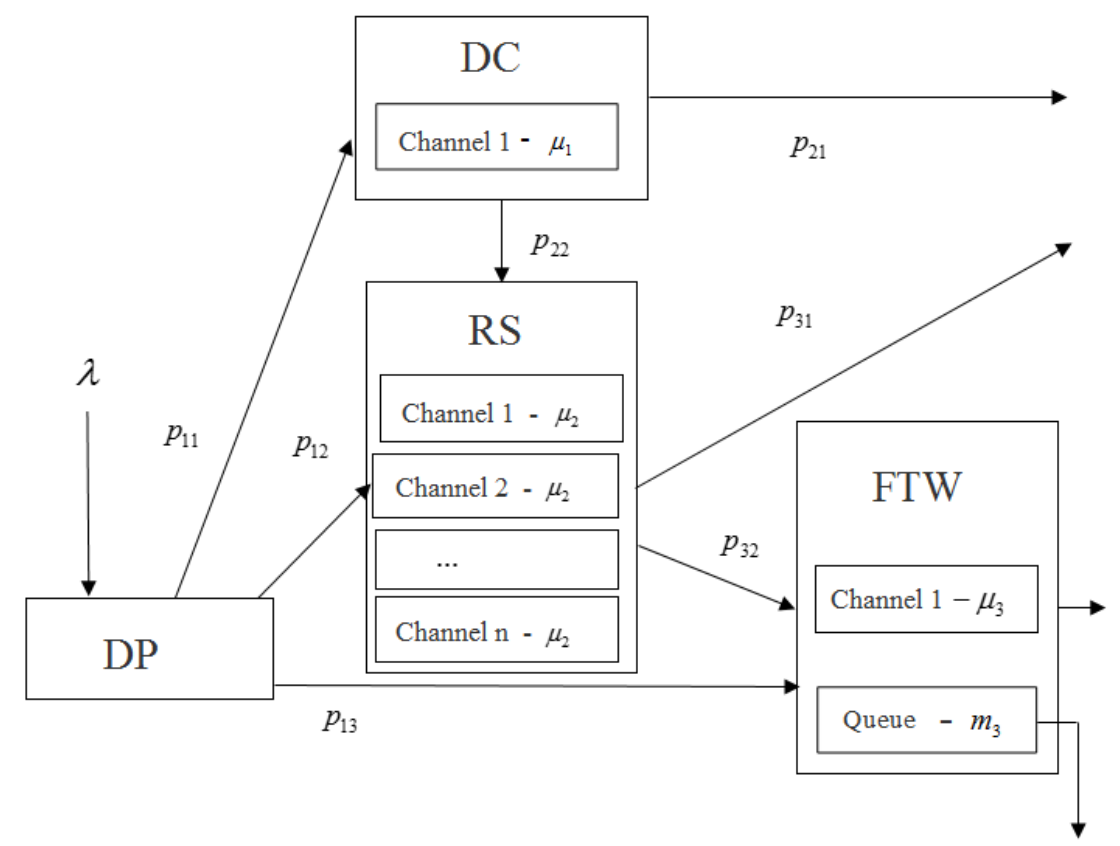

Fig. 1. Diagram of the maintenance center of ME.

DC - is a single-channel QS with expectation, unlimited queue length and bounded intensity of service. RS - is a multichannel QS with waiting, unlimited queue length and bounded intensity of each service channel. FTW - is single-channel QS with expectation bounded by a value of the queue length and bounded intensity of service. The complex, as a whole, is modeled as a three-phase QS.

From DC part of the stream goes out serviced and the other part goes to the RS. From RS part of the flow goes out serviced, and the other part goes to FTW. From FTW part of the flow goes out serviced, and a small part of the requirements leaves the system due to limited queue length.

Note that if the incoming stream on the DP is Poisson -stream, then the flows which directed to DC, RS, FTW are Poisson - streams too. If we assume that the service time of requirements in DC, PS, FTW is exponentially distributed random variable, then the outgoing RS and DC flows will also be Poisson - systems. Stream included in the RM and FTW are Poisson - streams too.

Note that flow of events coming out of FTW, strictly speaking, are not Poisson streams, since the stream of un served requirements has a form recurrence stream [4]. 
With other random laws that characterize the incoming flow of events and service time, the structure of the outgoing single-phase QS flows has a more complex probabilistic character. Such MQS can be investigated by means of simulation imitation methods [7].

\section{The mathematical model of a single-channel queueing system with expectation}

Let the incoming service request flow is the simplest, with intensity $\lambda$. Let the intensity of the flow of service $\mu$. Then service duration is a random variable subject to the exponential law of distribution. The service flow is the simplest Poisson - flow of events. A request received at the moment when the channel is busy, either becomes queued and waits for service if there are free places in the queue, or leaves the system unserviceable if all the places in the queue are occupied.

The stationary process in this system will be described by the standard system of algebraic equations [4].

Below are formulas for defining the characteristics of a single-channel QS with waiting and limited queue length equal to $m$ :

$P_{0}=(1-\rho) /\left(1-\rho^{m+2}\right)-$ the probability that there is no queue and the service channel is free, where $\rho=\lambda / \mu$ is the reduced intensity (density) of the flow,

$P_{k}=\rho^{k} P_{0}$ - the probability that one channel and $(k-1)$ places in the queue are occupied.

Note that compliance with the stationary condition $\rho=\lambda / \mu<1$ for a given QS is not necessary, since the number of applications entering the service system is controlled by imposing a limit on the queue length (which cannot exceed $m$ ), and not by ratio between the input and output intensities of flow $\rho$.

Denote:

$P_{o t k}=P_{m+1}=\frac{\rho^{m+1}(1-\rho)}{1-\rho^{m+2}} \quad$ - probability of denial of service request;

$q=1-P_{\text {otk }}$ - the relative throughput of the system;

$A=q \lambda-$ absolute throughput of the system;

$M[r]=\frac{\rho^{2}\left(1-\rho^{m}(m+1-m \rho)\right)}{\left(1-\rho^{m+2}\right)(1-\rho)}$ - average number of request in queue;

$M[n]=0 \cdot P_{0}+1 \cdot\left(1-P_{0}\right)=\frac{\rho-\rho^{m+2}}{1-\rho^{m+2}}-$ average number of request under service;

$M[s]=M[r]+M[n]$ - average number of request in the system (related to QS);

$T_{s}=T_{w}+t_{o b}$ - average time of request stay in the system;

$T_{w}=M[r] /(\rho \mu)=M[r] / \lambda$ - average length of stay of the client (request) in the queue.

\section{The mathematical model of the multi - channel queueing system with a waiting queue}

The probability characteristics of functioning in the stationary mode, multi-channel QS with the waiting queue determined by the following formulas:

$$
P_{0}=\left(\sum_{k=0}^{n} \frac{\rho^{k}}{k !}+\frac{\rho^{n+1}}{n ! \cdot(n-\rho)}\right)^{-1} \text { - the probability that all channels of service are free (for QS }
$$

with unlimited queue length); 


$$
P_{0}=\left(\sum_{k=0}^{n} \frac{\rho^{k}}{k !}+\frac{\rho^{n+1}}{n ! \cdot n} \cdot \frac{\left(1-\rho^{m}\right)}{(1-\rho)}\right)^{-1}-\text { the probability that all channels of service are free (for QS }
$$

with limited-size queue length);

$$
P_{o t k}=P_{m+n}=\frac{\rho^{n+m}}{n^{n+m} \cdot n !} P_{0} \text { - the probability of failure of the system (all channels of service }
$$

and places in the queue are busy);

$P_{\kappa}=\frac{\rho^{k}}{k !} P_{0} \quad-$ the probability that an occupied all service channels and all places in the queue are free;

$P_{n+r}=\frac{\rho^{n+r}}{n^{r} \cdot n !} P_{0}$ - the probability that $k$ service channels are busy and $r$ places in the queue are free;

$M[r]=\frac{\rho^{n+1} P_{0}}{n \cdot n !} \cdot \frac{1-(m+1) \chi^{m}+m \chi^{m+1}}{(1-\chi)^{2}}-$ average number of requests per queue,

where $\chi=\frac{\rho}{n}$;

$M[n]=\rho\left(1-\frac{\rho^{n+1} P_{0}}{n^{m} \cdot n !}\right)$ - average number of busy channels;

$M[s]=M[r]+M[n]$ - average number of requests in the system;

$T_{w}=\bar{r} / \lambda \quad$ - average queue waiting time for an order;

$t_{s}=T_{w}+1 / \mu$ - average length of stay in the system.

\section{Statement of the problem the distribution of the manufacturing capacities}

Let us consider the problem of rational distribution of production and technical capacity between RS and FTW. It is assumed that part of the production and technical capacities of RS can be transferred to FTW, attached to FTW and used as production and technical capacities of FTW. As the controlled parameter we will choose reference unit production capacity transmitted from the RS in FTW. We supposed that In the case of absence of places in the queue in FTW request leaves the system without unserviceable.

The following two mutually complementary optimal control problems [8] were investigated in the paper:

1. The problem of the minimum number of requirement leaving FTW unfulfilled for different values of the restriction on the total stay time of the requirement in MQS.

2. The problem of minimum total service time of requests to the MQS for a given upper restriction on the proportion requests leaving the MQS unfulfilled requirement.

\section{Method of solution}

The method of sequential linearization [8], which is a direct approximate optimization method, was chosen as a basic method.

The parameters of the real existing QS were selected as the first approximation when searching for optimal control. At each step of the iterative process, an improving sufficiently small addition $\delta u_{i}$ to the control parameter $u_{i}$ and an appropriate improved 
control $u_{i}=u_{1}+\delta u_{i}$ was found. This procedure should ensure the minimum value of the criterion and fulfillment of all restrictions in the linear approximation.

Calculations have shown that after 10-20 iterations the process of stabilization of the solution with respect to a certain set of parameters occurs. This may be both evidence that an approximate optimal solution has already been found and evidence that the method used at this stage of optimization has become ineffective.

Therefore, to confirm the correctness of the obtained results (approximate optimality) for each optimization problem calculations were carried out using different initial approximations for the desired parameters. All calculations provided the same result. The coincidence of the results obtained using different initial approximations gives reason to believe that the results obtained are correct.

\section{The results of calculation}

Calculations were carried out at the following values of parameters of QS: $\lambda=8, p_{11}=0,12$, $p_{12}=0,07, p_{13}=0,81, p_{21}=0,75, p_{22}=0,25, p_{31}=0,81, p_{32}=0,19, \quad \mu_{1}=2, \mu_{2}=3, \mu_{3}=2$, $m_{3}=2$.

Figure 2 shows the dependences for RS: the average number of requests in the queue, the average number of requests in system and the average number of free channels on the number of units of production capacity transferred from RS to FTW are presented.

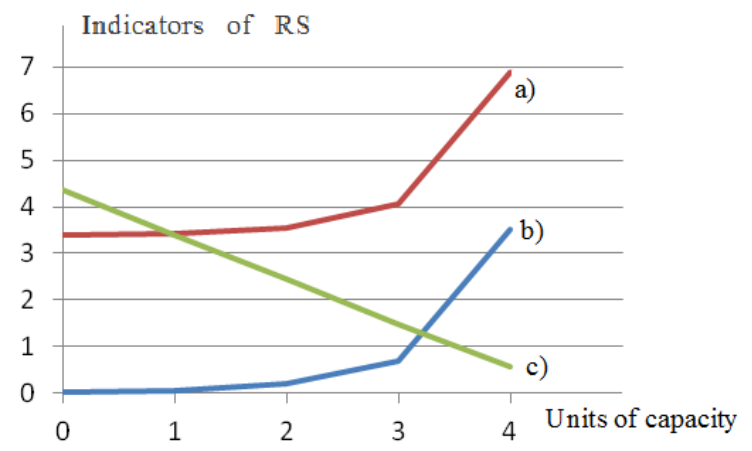

Fig. 2. The dependence of indicators for RS from the number of transmitted units of capacity: a) the average number of requests in the queue; b) the average number of requests in the system; c) the average number of free channels.

Figure 3 presents the dependence for FTW: average number of requests in the queue, the average number of requests in system and the proportion of requests that have left FTW. It can be seen that with the increase in the transferred units of production capacity, the RS indicators deteriorate somewhat, and the indicators of FTW are significantly improved. However, the technical and production performance of MQS as a whole is improving. For example, the share of outgoing requests from FTW significantly decreases from 0.22 to 0.005 with an increase in the average queue time of the application from 0.37 to 0.54 . 


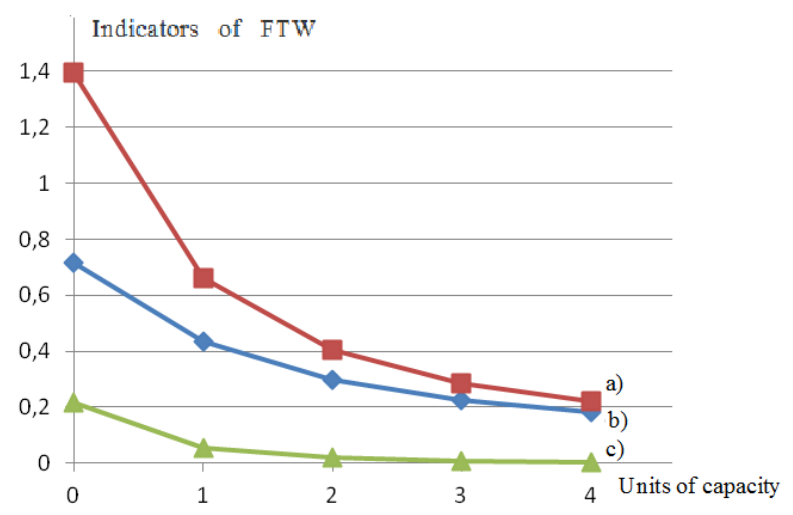

Fig. 3. The dependence of indicators for RS from the number of accepted units of capacity: a) the average number of requests in the system; b) the average number of served requests; c) share of requests outgoing from FTW.

\section{Conclusion}

Carried out adaptation of the method of successive linearization to the problem of optimizing the operation of the MQS. Developed specialized software that allows you to simulate and optimize the operation of the MQS. Approbation of the method for the simplest of the incoming stream and the flows generated by simulation using the method of consistent conduct transactions was carried out.

The results of the analysis of the indicators that characterize the quality of the functioning of the MQS allow to identify both the strengths and weaknesses of the MQS. According to the results of the analysis can be determined the direction of improvement in specific indicators of the MQS.

\section{References}

1. P.A. Marichev, A.S. Kornev, R.Z. Khayrullin, Vestnik MGSU, 13, 71 (2018)

2. R.Z. Khayrullin, MATEC Web Conf., 117, 76 (2017)

3. R.Z. Khayrullin, MATEC Web Conf., 86, 5018 (2016)

4. E.S. Ventzel, Operations Research (Moscow, Science Pub, 1972)

5. V.V. Grachev, A.N. Moiseev, A.A. Nazarov, V.Z. Yampolsky, Proceedings of TUSUR, 2 (2012)

6. I. N. Bojarshinova, T.R. Ismagilov, I.A. Potapova, Fundamental research, 9-1 ( 2015)

7. T. Altiok, B. Melamed, Simulation Modeling and Analysis with ARENA (Amsterdam, Boston, Academic Press, 2007)

8. R. P. Fedorenko, Approximate solution of optimal control problems (Moscow, Science Pub, 1978) 